\title{
A UTILIZAÇÃO DOS RECURSOS EDUCACIONAIS DO NI MULTISIM LIVE E PSIM PARA A INTRODUÇÃO DE SIMULAÇÕES EM ELETRÔNICA DE POTÊNCIA
}

Flávio Fernando França Farias-ffff@discente.ifpe.edu.br Instituto Federal de Pernambuco

R. Padre Agobar Valença, s/n - Severiano Moraes Filho 55299-390 - Garanhuns - Pernambuco

Hemilly Sara Plácido de Lima-hspl@discente.ifpe.edu.br Instituto Federal de Pernambuco

R. Padre Agobar Valença, s/n - Severiano Moraes Filho 55299-390 - Garanhuns - Pernambuco

Jaiannydos Santos Cabral-jsc3@discente.ifpe.edu.br Instituto Federal de Pernambuco

R. Padre Agobar Valença, $s / n$ - Severiano Moraes Filho 55299-390 - Garanhuns - Pernambuco

Romana Emanuelle da Silva Galdino - resg@discente.ifpe.edu.br Instituto Federal de Pernambuco

R. Padre Agobar Valença, s/n - Severiano Moraes Filho 55299-

390 - Garanhuns - Pernambuco

Kleber Paulo da Silva - kleberpaulo@hotmail.com

Instituto Federal de Pernambuco

R. Padre Agobar Valença, s/n - Severiano Moraes Filho

55299-390 - Garanhuns - Pernambuco

Mário Jorge Guimarães Rocha Neto - mario.neto@garanhuns.ifpe.edu.br Instituto Federal de Pernambuco

R. Padre Agobar Valença, s/n - Severiano Moraes Filho

55299-390 - Garanhuns - Pernambuco

Resumo: A crescente demanda exigida pelo mercado de trabalho na área da eletrônica de potência leva instituições de ensino a investirem em laboratórios de ponta, que possuem preços onerosos no mercado. Enveredar essa disciplina pelos caminhos laboratoriais vem sendo uma metodologia utilizada para o ensino e o aprendizado desse componente curricular, buscando corroborar o binômio teórico-prático. Sendo assim, o presente artigo trata da estratégia pedagógica de levar o ensino da eletrônica de potência a patamares experimentais, baseandose em softwares de simulação, como PSIM e NI Multisim, que simulam um ambiente laboratorial, proporcionando aos seus usuários simular possíveis situações. Para tanto, os processos de revisão bibliográfica seguidos de simulações e finalizados com as práticas alicerçam a metodologia adotada. Dessa forma, os resultados indicaram uma ascensão gradativa na curva de aprendizado dos alunos os quais foram inseridos nesse método. 
"Os desafios para formar hoje o engenheiro do amanhã"

Palavras-chave: Eletrônica de Potência. Teórico-prático. Softwares. Simulação.

\section{INTRODUÇÃO}

A quantidade de processamento e compartilhamento no fluxo de informações, possibilitada através do aperfeiçoamento dos transistores, permitiram um exponencial desenvolvimento de circuitos eletrônicos, sobretudo os integrados, tendo como uma das aplicações de destaque, o uso nos processadores de computador. Com isso, o processo de globalização desenvolvido fortemente pela a expansão dos sistemas de: comunicação, informática, transportes e telefonia, proporcionaram uma intensificação das relações socioeconômicas entre os diversos países do mundo (MUNDO EDUCAÇÃO, 2020).

Indubitavelmente, tais transformações refletem no processo educacional, desde os níveis mais básicos da educação ao superior, ocorrendo gradualmente a inclusão da tecnologia na forma de ensino, haja vista, ao atual processo em desenvolvimento, o da quarta Revolução Industrial, conhecido como Indústria 4.0, o qual "promove a informatização da indústria e tem como base as inovações tecnológicas, permitindo a conectividade da automação, do controle e da tecnologia da informação para aprimorar os processos da manufatura e sua eficiência." (OLIVEIRA; SIMÕES, 2017). Dessa forma, o mercado de trabalho já exige dos novos profissionais uma formação multidisciplinar e flexível, como também, o domínio de novas ferramentas, para que o indivíduo seja capaz de adaptar-se a novas modalidades de gestão e controle remoto de processos (ALESSANDRO NUNES, 2018).

Em consequência disso, os computadores são fortes aliados à educação e ao trabalho científico, uma vez que impasses na engenharia na grande maioria das vezes são resolvidos ou evidenciados por meio de simulações de projetos, que por sua vez auxiliam na prevenção de erros ou acidentes, reduzindo custos materiais e humano. Como também, simulações computacionais atuam como ferramenta e técnica didática para propiciar uma interdisciplinaridade da disciplina lecionada.

Destarte, diversos projetos e estudos foram elaborados de modo a amplificar e analisar o uso da simulação de circuitos conjuntamente com a disciplina estudada pelos discentes, como Gomes et al. (2012), apresentou um software em que o aluno pode simular circuitos normalmente utilizados no estudo da Eletrônica de Potência, mostrando através deste ambiente, os resultados para a situação simulada pelo usuário. Sobretudo, o apresentado por Brioschi (2012), em que após a aplicação de softwares no ensino da Eletrônica de Potência, demonstrou que houve uma melhora na aprendizagem dos conteúdos da disciplina e do desempenho dos alunos. Considera-se a preparação de aplicações em Eletrônica de Potência como um trabalho complexo, devido ao caráter experimental e multidisciplinar desta ciência (FERREIRA et al, 2005).

Por essa razão, este trabalho tem como propósito habituar previamente os alunos envolvidos com a disciplina de eletrônica de potência, em ambientes laboratoriais de simulação, utilizando os recursos educacionais do NI Multisim Live e do PSIM, para a introdução em simulação de circuitos que possuem cargas resistivas, capacitivas e indutivas, aliados à prática e o funcionamento do controle de potência destes, de modo online e remoto. De forma que os discentes recém inseridos nesta disciplina, possam ver, de antemão, estes circuitos funcionando de forma completa, economizando tempo em parâmetros, servindo como um pré-experimento de tais. Bem como, estimular os discentes a desenvolverem uma visão sistêmica, estratégica e independente, materializando uma 
"Os desafios para formar hoje o engenheiro do amanhã"

postura construtiva, e potencializando a aprendizagem, através destas ferramentas de simulações.

\section{FUNDAMENTAÇÃO TEÓRICA}

Nesta seção são apresentados os softwares escolhidos para a elaboração deste projeto, tal como, são evidenciados os trabalhos de ensino encontrados na literatura acadêmica que auxiliaram na elaboração desta produção educacional, voltada para a ambientação inicial dos discentes, com estes simuladores relacionados à Eletrônica de Potência.

\subsection{Multisim NI e PSIM}

O Multisim desde a sua criação mantém o gérmen educativo. O sistema operacional foi especificado pela empresa para ser um ambiente de simulação SPICE (Simulation Program With Integrated Circuit Emphasis), com padrões industriais esquemáticos e interface intuitiva, permitindo a visualização e análise do funcionamento de componentes eletrônicos, por meio de simulação, como também, a aplicabilidade em projetos de ensino e pesquisa nas áreas de eletrônica digital, analógica e de potência (MULTISIM, 2020). Dessa forma, o NI Multisim permite uma proficiência no entendimento sobre conceitos teóricos vistos durante o curso de engenharia, nas matérias de Circuitos elétricos, eletrônica de potência, eletrônica (analógica e digital), mecatrônica e outras ciências que utilizam-se de circuitos elétricos, de forma aliada à simulação de circuitos, permitindo em momentos críticos o ensino remoto, sendo vastamente aplicado nas instituições de ensino técnico e superior da área elétrica.

Dessarte, o NI Multisim for Education é uma divisão da National Instruments, que possui uma versão do software NI Multisim estudantil, a qual possibilita a análise do comportamento de circuitos, o ensino da eletrônica e a comparação teórica entre sinais reais e simulados (MULTISIM, 2020). Do mesmo modo, é disponibilizada uma versão do sistema operacional online e gratuita, o NI Multisim Live, voltada para a aplicação e o ensino de circuitos elétricos básicos para cursos e laboratórios de eletrônica analógica, digital e de potência, de modo inicial, após cadastro na plataforma.

O PSIM é um sistema operacional desenvolvido pela empresa Powersim, utilizado para simulação de qualquer circuito elétrico, seja digital ou analógico, sobretudo, projetado para a utilização em eletrônica de potência e acionamento de motores elétricos, possuindo uma simulação precisa e veloz. Este software é capaz de simular conversores em eletrônica e controle, possuindo em sua bagagem modelos específicos de chaves estáticas (PSIM, 2020).

Desse modo, é um excelente recurso didático e científico, possibilitando o uso deste como ferramenta fundamental para estes fins. Sua interface é de fácil compreensão e intuitiva, fornecendo a captura esquemática e a visualização de formas de ondas, por meio do Simview, onde os seus componentes eletrônicos têm variados níveis de complexidade, começando com comportamento e características ideais para não ideais. Utiliza, como base do seu algoritmo de simulação a análise nodal juntamente com a integração de regras trapezoidais, resolvendo equações simultâneas durante o seu uso em simulação, não gerando matrizes de espaço de estado, que por vezes, apresentam problemas de convergência em grandes derivadas (PSIM, 2020).

Além disso, o PSIM dispõe de diversos módulos que amplificam a sua funcionalidade em simulação e design de circuitos como os de: motores elétricos, energia fotovoltaica, turbinas eólicas e térmicas, sendo este último, capaz de simular e calcular as perdas de 
comutação e condução térmicas de dispositivos como MOSFET (Transistor de Efeito de Campo de Óxido de Metal Semicondutor), IGBTS (Transistor Bipolar de Porta Isolada), diodos e chaves GaN (transistores de nitreto de gálio), determinando as características de perda (PSIM, 2020).

\subsection{Literatura Acadêmica}

A Eletrônica de Potência é a combinação entre eletrônica, potência e controle, podendo ser estabelecida como a aplicação da eletrônica para o controle e conversão da energia elétrica (RASHID, 1999). Uma ciência importante no desenvolvimento tecnológico.

Com isso, resultando na popularização dos computadores a partir de meados dos anos 90, alavancando a presença destes dispositivos em diversos segmentos, desde o industrial ao pessoal. Em razão disso, a necessidade de adaptação e manuseio desta ferramenta, se fez indubitavelmente presente nos últimos vinte anos, de forma a otimizar as funcionalidades dos recursos computacionais oferecido por estas máquinas (SANTOS et al., 2012).

Sendo assim, o modo de ensino denominado como Pedagogia da Transmissão, que consiste na prática habitual do docente de propagar ideias e conhecimentos referentes à matéria lecionada, como ponto alto da metodologia de ensino, tendo o aluno como um componente passivo, onde lhe cabe o preceito de receber e reproduzir o que lhe é perpassado (BORDENAVE, 1983), está ficando obsoleto. Tendo em vista, as inovações constantes requeridas pelo mercado de produção, a julgar pelo processo de adaptação às novas características da indústria 4.0.

Segundo Bianchini e Gomes (2007) ensinar com o auxílio de softwares procurando imitar cenários reais, ajuda a transpassar a separação entre teoria e compreensão, intensificando o entendimento ao estudar, oferecendo ao discente uma oportunidade para contextualizar o conhecimento e incorporá-lo quando precisar aplicá-lo. Portanto, segundo o que Huczynski e Johnston (2005) relataram, o uso de componentes computacionais estimula um comportamento autônomo do aluno em aprender, desta forma, ajudando na construção de uma postura mais construtiva do estudante de engenharia (MASSUKADO; SCHALCH, 2007).

\section{DESENVOLVIMENTO DO PROJETO}

Nesta seção, serão relatadas todas as etapas gradativas da metodologia do projeto, desde a revisão bibliográfica até o processo de montagem, com a ressalva da monitoria em turmas do nível técnico que possuem em sua grade curricular a disciplina Eletrônica de Potência.

\subsection{Revisão bibliográfica}

Conforme foi dito na literatura acadêmica, algumas literaturas na área de ensino da eletrônica de potência foram tomadas como base. Dessa forma, uma leitura minuciosa foi efetuada para o embasamento teórico, com a finalidade de construir um acervo de dados que, futuramente, nas etapas de simulação e montagem, pudessem ser confirmados.

\subsection{Simulação}

A etapa de simulação a ser inserida como processo de ensino-aprendizado na engenharia como um todo não é uma metodologia inovadora. No entanto, esse caminho vem sendo adotado gradativamente por componentes curriculares como eletrônica de potência, que precisam de um alto investimento para que seus laboratórios sejam adquiridos. Dessa 
forma, um ambiente remoto passa a ser uma das soluções adotadas, e exigidas, em muitos casos.

Sendo assim, baseando-se no método de antecipar a prática por meio das simulações, os discentes foram inseridos nesse processo simulatório. A princípio, softwares como NI Multisim e Psim suprem todas as necessidades básicas e introdutórias das demandas.

\subsection{Monitoria e Prática}

O processo de monitoria foi tomado como estratégia pelos envolvidos do projeto, para que os discentes responsáveis pelo mesmo pudessem entrar em contato com pessoas que estivessem cursando o componente curricular Eletrônica de Potência, e, adicionado, ocorressem trocas de ensinamentos e eventuais imbróglios no decorrer do processo de aprendizado. Bem como, analisar qual a melhor linguagem a se adotar para ensinar a disciplina, e a intelecção de uma das principais dificuldades dos alunos, que é o espaço que existe entre a compreensão do funcionamento das chaves semicondutoras e conversores de potência, de modo teórico, até a sua visualização prática em laboratório.

Para tanto, o orientador, que também foi o docente responsável pelo processo de ensino e aprendizado dessa disciplina nas turmas do terceiro período do nível técnico no ano de 2018, destinava às práticas aos projetistas, para que os mesmos pudessem estudar e até mesmo simular e montar os circuitos antes delas serem realizadas, com a finalidade de estarem preparados para possíveis questionamentos e eventuais erros que viessem à tona.

De todas as etapas, o processo de práticas em laboratório é de suma importância para a construção profissional e acadêmica do aluno. Além do mais, as experiências que vão sendo adquiridas, assim como a confiança gerada após o término de cada simulação e montagem não devem servir de esquecimento para as revisões bibliográficas estarem em dia. Isto é, os processos de leitura, simulação e montagem devem ser concomitantes, para a concretização da metodologia.

\section{RESULTADOS}

Ainda nas primeiras práticas da disciplina de Eletrônica de Potência, percebeu-se que os discentes estavam tendo dificuldades, por exemplo: não estarem familiarizados com os simuladores e suas funções, o que acarretava em recorrentes dúvidas acerca da montagem e do funcionamento de uma das topologias iniciais para os estudos dos retificadores, o retificador de meia onda não controlado, cujo objetivo é ajustar o valor da tensão saída, transformando em um sinal CC. Conforme está sendo mostrada a sua simulação no software PSIM, na Figura 1, composta por uma fonte de tensão alternada de $311 \mathrm{~V}$, um diodo, uma carga RL, formada por um indutor de $50 \mathrm{mH}$ e um resistor de 270 Ohms. Como também, as suas respectivas formas de onda de tensão, na Figura 2.

Figura 1 - Retificador de meia onda não controlado simulado no PSIM

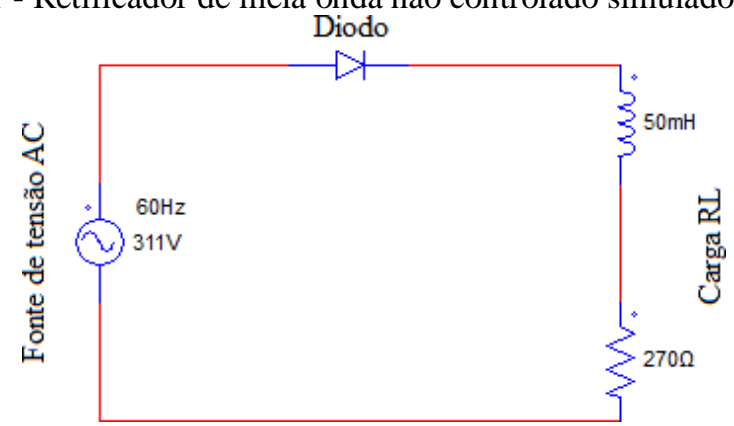

Fonte: os autores 
A Figura 2, logo abaixo, demonstra as formas de onda da tensão, em volts, ao longo do tempo, em segundos, do diodo (em a) e do resistor (em b). A partir dos gráficos obtidos pela simulação, nota-se a retificação da tensão na carga RL, ver Figura $2 b$, com o diodo conduzindo apenas no semiciclo positivo da tensão de entrada CA, sendo o semiciclo negativo da tensão de entrada removido na saída. Durante o processo de corte do diodo, dado que a tensão na carga é zero, toda a tensão de entrada presente no semiciclo negativo deverá ser suportada pelo semicondutor, como visto na Figura 2a. Tais resultados, caso coletados nos momentos que antecederem os experimentos laboratoriais, poderão reduzir a distância, por vezes, impeditiva de compreensão dos temas correlatos, entre a teoria e a prática nos cursos técnicos e superiores que possuam competências voltadas para Eletrônica de Potência.

Figura 2 - Formas de onda do retificador de meia onda não controlado

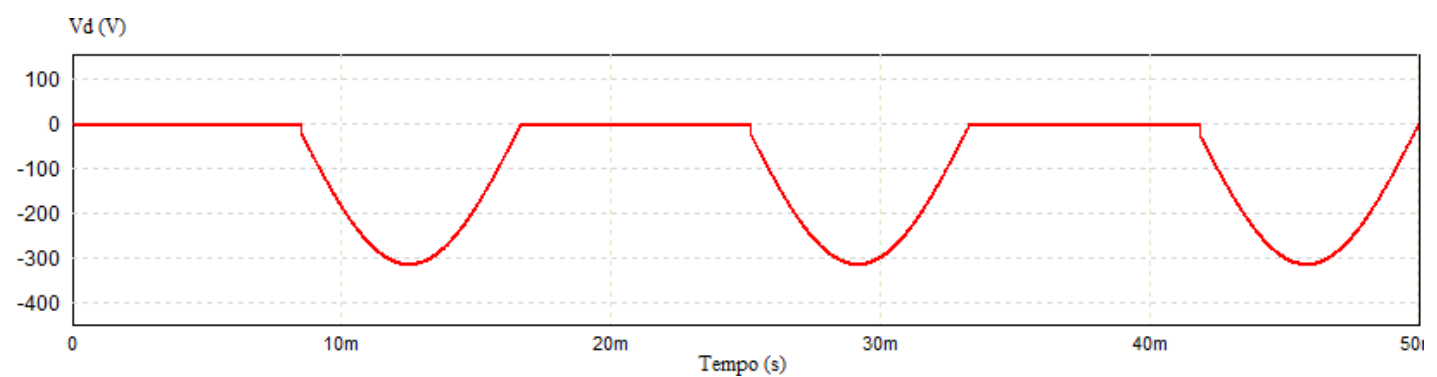

(a)

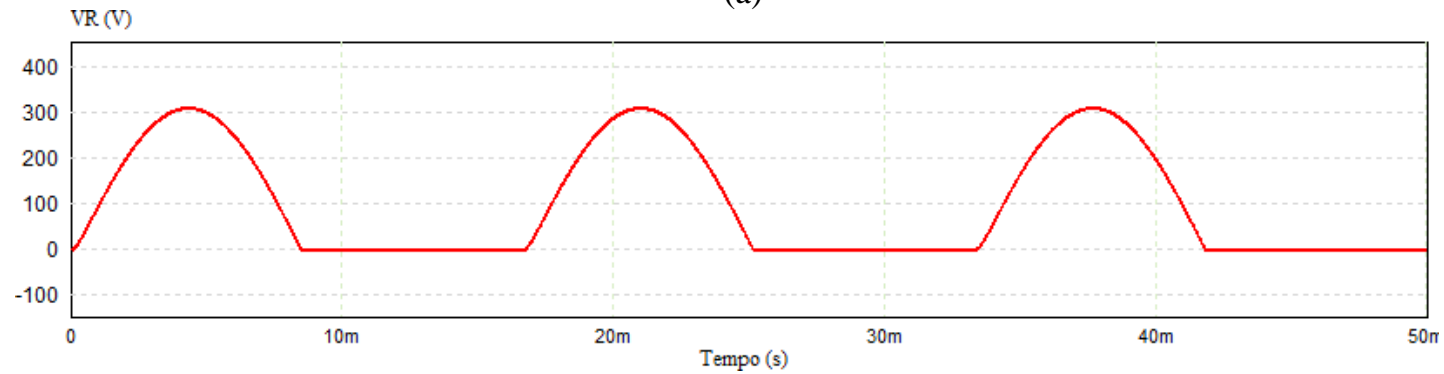

(b)

Fonte: os autores

Na plataforma de simulação Multisim Live, foi reproduzido um conversor CC do tipo buck (circuito elétrico abaixador de tensão contínua), ver Figura 3. Nesta configuração, a chave semicondutora S1 (MOSFET) entrega pulsos elétricos a um circuito formado por um indutor (L1), capacitor (C1) e um diodo (D_ideal), possibilitando, dessa forma, a obtenção de uma tensão contínua na carga. Através da técnica de modulação por largura de pulso (PWM), o circuito de controle U1 poderá controlar o nível de tensão contínua na carga, para valores entre $0 \mathrm{~V}$ e a fonte de tensão V1. As formas de ondas da tensão e corrente na carga, apresentadas na Figura 4, foram obtidas a partir de uma frequência de chaveamento de $100 \mathrm{kHz}$ e ciclo de trabalho de $20 \%$. Para as formas de onda de tensão e corrente na carga, percebe-se que, ao acionar o conversor, antes de atingir o regime permanente, surge uma tensão de pico de, aproximadamente, duas vezes o valor da tensão contínua estimada pelo circuito de controle. É uma fase transitória importante a ser observada, uma vez que, ao analisar graficamente os resultados simulados, o aluno tem a percepção dos riscos envolvidos nas práticas laboratoriais e nos projetos. Adicionalmente, demonstra também a necessidade do correto dimensionamento dos componentes e dos circuitos de proteção presentes nos conversores CC. 


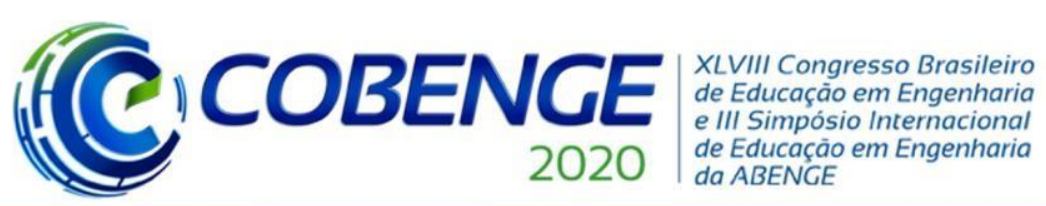

"Os desafios para formar hoje o engenheiro do amanhã"

Figura 3 - Simulação do Conversor Buck no Multisim Live

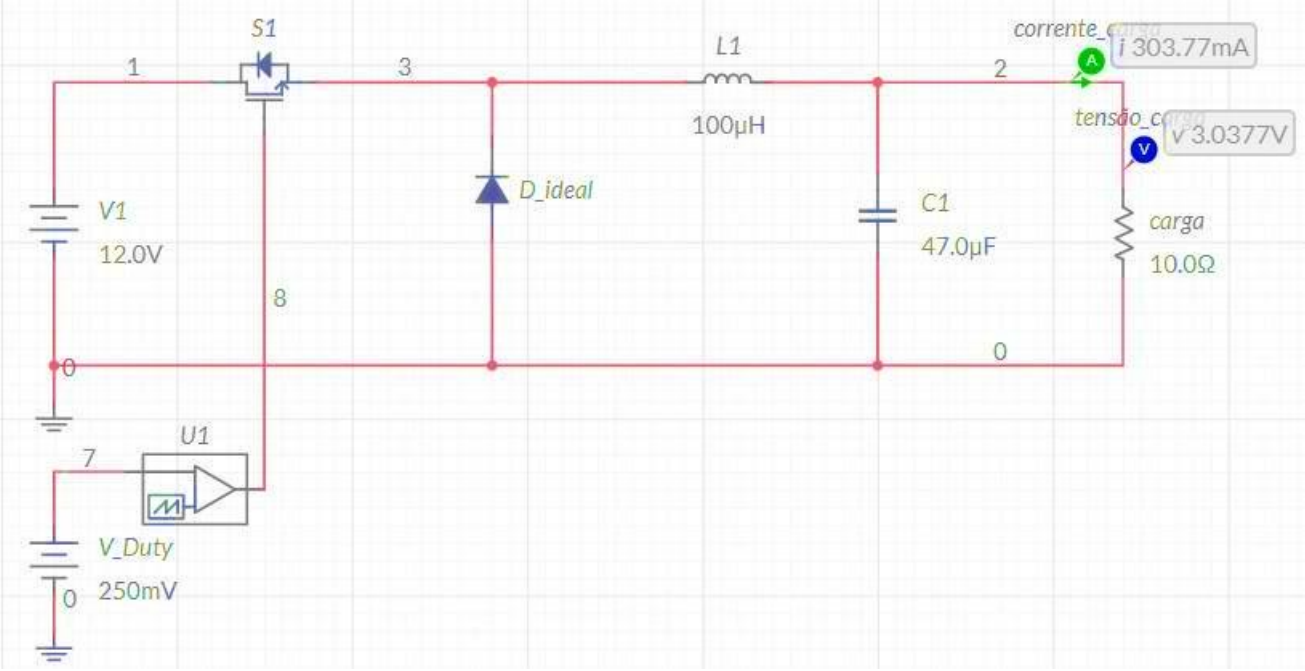

Fonte: os autores

Figura 4 - Formas de ondas da tensão e corrente na carga do conversor Buck via Multisim Live

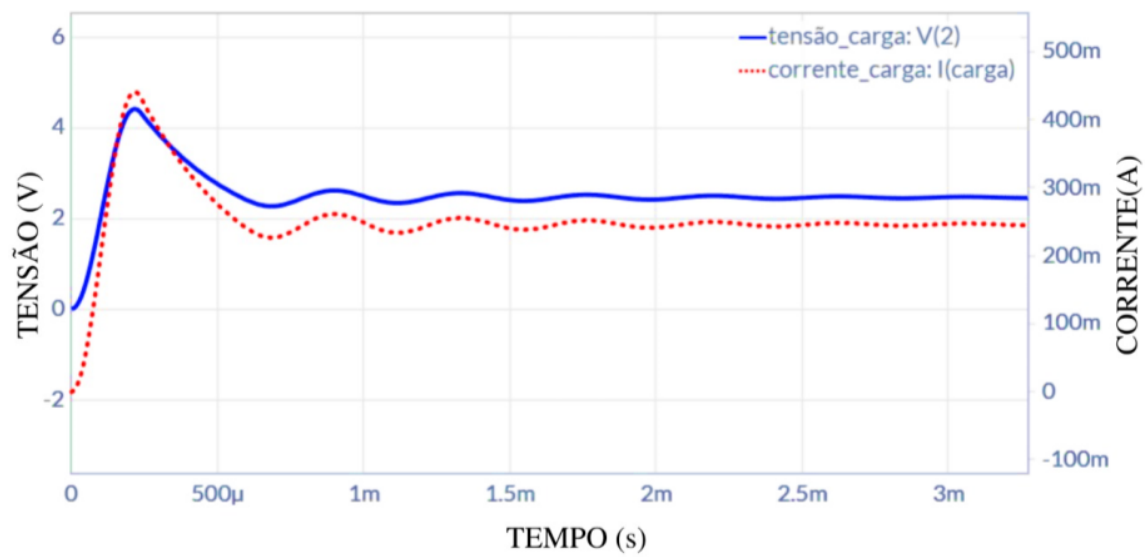

Fonte: os autores

Com a utilização do PSIM e Multisim Live como ferramenta auxiliar de ensino, o uso de simuladores contribui para melhorar a curva de aprendizagem dos alunos nesta disciplina. A partir destes programas, é possível gerar formas de onda de tensão e corrente, valores de pico, de potência, de potência média, valor RMS, analisar o regime transitório e permanente, que todo conversor passa. De modo que, é possível ver anteriormente o conversor funcionando de forma completa, economizando tempo em parâmetros e servindo como um pré-experimento. Além de possibilitar uma visão mais transparente da disciplina de forma computacional, que não é possível apenas de modo teórico.

Como comprovação da veracidade desta melhoria, notou-se através da elaboração de questionários avaliativos que, quando aplicada a metodologia de ensino nos ambientes de simulação, resultados satisfatórios são obtidos pelo público, tanto na compreensão do conteúdo abordado, quanto na prática, sendo o medidor crucial deste método, a abordagem do mesmo em um minicurso de simulação através de ambientes virtuais. 


\section{CONSIDERAÇÕES FINAIS}

O alto custo dos laboratórios e bancadas didáticas de eletrônica de potência, aliado com a crescente demanda exigida pelo mercado de trabalho nessa área, leva instituições de ensino a tomarem providências quanto a sua metodologia adotada. Ao se depararem com o método chamado pedagogia transmissiva, em que o aluno não interage e é tomado como indivíduo passivo do processo educacional, encontram um gargalo.

Dessa forma, dentre diversas soluções, uma que vem sendo bastante implementada e dando resultados, é a simulação seguida de montagem. Para tanto, softwares como PSIM e NI Multisim aparecem como um intermédio entre a teoria e prática, permitindo que os estudantes visualizem, de maneira mais facilitada, os conteúdos vistos em sala de aula ao comparar com os resultados obtidos via simulação, além de possibilitar análise de grandezas e fazer estimativas antes da implementação, de fato, de um sistema, com a finalidade de evitar possíveis erros. E tudo isso sem a necessidade de um aparato de instrumentos, a qualquer hora e em qualquer lugar sem comprometer a integridade dos aparelhos e adquirindo conhecimento de maneira semelhante, porém com muito mais praticidade. Além da liberdade proporcionada por estes, a facilidade do manuseio, a comodidade oferecida, a biblioteca de componentes, a não exigência do download por uns, são pontos positivos que fazem a ideia da utilização de simuladores, no processo de aprendizagem, uma ferramenta interessante.

Destarte, o projeto aliou o processo de revisão bibliográfica com o de simulação e montagem, o que facilitou a identificação dos eventuais erros que viessem a ocorrer, assim como saber lidar com determinadas situações e ir em busca de soluções. Uma base bem consolidada juntamente com a metodologia mencionada anteriormente, simulação seguida de montagem, levam a construção de um discente que atenda, gradativamente, às exigências que o mercado de trabalho vem cobrando.

Em resumo, depreende-se que o processo de ensino-aprendizado da Eletrônica de Potência vem sendo modificado, nos últimos anos, conforme exigido pelo ambiente que tais discentes serão inseridos, no qual a qualidade de um serviço fornecido com menor custo possível é um fator determinante para os futuros profissionais da engenharia. Pelo arranjo atual da sociedade, no qual o sistema de ensino remoto está sendo cada vez mais adotado ou então os recursos no laboratório não são suficientes para atender as demandas do componente curricular, a ideia de aliar a simulação ao ensino de engenharia é cada vez mais necessária e urgente, podendo ser implementado sem muitas complicações, originando, deste modo, um laboratório virtual no qual o docente seria capaz de acompanhar o desempenho dos estudantes sem muitas dificuldades. Essas mudanças potencializam a busca por informações e solução do problema em questão, confrontando os mesmos, levando-os a confirmar, em partes, a teoria na prática.

Portanto, como perspectiva futura, a realização de minicursos com o cunho informativo de assuntos básicos, a princípio, deve ser efetuada para reparar possíveis falhas do ensino básico. Assim, por meio desses ajustes, os alunos possam voltar a tecer a curva de aprendizado, minimizando os eventuais problemas. E, posteriormente, de forma compassada, adentrar em assuntos mais complexos. 


\section{REFERÊNCIAS}

ALESSANDRO NUNES (Brasil). Computerworld. Quais competências o profissional da indústria 4.0 deve ter? 2018. Disponível em

https://computerworld.com.br/2018/07/27/quais- competencias-o-profissional-da-industria-40-deve

ter/\#: :text=Quem\%20\%C3\%A9\%20o\%20profissional\%20da,socialmente\%20para\%20dese mpenhar\%20trabalhos\%20colaborativos.Acesso em: 17 jul. 2020.

BIANCHINI, David; GOMES, Francisco de Salles C. A SIMULAÇÃO COMO FERRAMENTA DIDÁTICA NO ENSINO DE ENGENHARIA. In: XXXV CONGRESSO BRASILEIRO DE EDUCAÇÃO EM ENGENHARIA, 2007, Curitiba-PR. Anais. CuritibaPR, 2007. Disponível em: http://www.abenge.org.br/cobenge/arquivos/12/artigos/368David\%20Bianchini.pdf. Acesso em: 20 jul. 2020.

\section{BORDENAVE, Juan E. Dias. ALGUNS FATORES PEDAGÓGICOS. Revista}

Interamericana de Educação de Adultos, Brasil, v. 3, 1983. Disponível em: http://www.fo.usp.br/wp-content/uploads/EAlguns.pdf. Acesso em: 17 jul. 2020.

BRIOSCHI, Ricardo D. O. INOVAÇÃO EDUCACIONAL NO ENSINO DA ELETRÔNICA DE POTÊNCIA COM RECURSOS DE MULTIMÍDIAS: PRODUÇÃO E

IMPLICAÇÕES NA PRÁTICA EDUCATIVA. In: Congresso Brasileiro de Educação em Engenharia, 2012. Anais. Disponível em: http://www.abenge.org.br/cobenge/arquivos/5/Artigos/128870.pdf. Acesso em: 20 jul. 2020.

FERREIRA, A.A. et al. Integrated Platform for Power Electronics Applications Fast Evaluation and Teaching Purposes. Anais: IEEE Power Electronics Education Workshop, PEEW. Recife, Brasil. p. 81-86, 2005.

HUCZYNSKI, A.; JOHNSTON, S. P. Engineering students' use of Computer Assisted Learning (CAL). European Journal of Engineering Education, v. 30, n. 2, p. 287-298, 2005. Disponível em: https://www.tandfonline.com/doi/abs/10.1080/03043790500087530. Acesso em: 20jul. 2020.

MASSUKADO, Luciana Miyoko; SCHALCH, Valdir. Simulação no Ensino de Engenharia - Avaliando a Aplicação do Software Simgere sob o Paradigma do "Aprender a Aprender". Revista de Ensino de Engenharia, [S.I.], v. 26, 40-46, 2007. Disponível em: https://www.researchgate.net/publication/267561173_Simulacao_no_Ensino_de_Engenharia _Avaliando_a_Aplicacao_do_Software_Simgere_sob_o_Paradigma_do_Aprender_a_Apren der. Acesso em: 20 jul. 2020.

MULTISIM. Disponível em: https://www.ni.com/pt-br/shop/electronic-testinstrumentation/application-software-for-electronic-test-and-instrumentation-category/whatis-multisim.html. Acesso em: 17 jul. 2020.

MUNDO EDUCAÇÃO (Brasil). O que é Globalização? Disponível em: https://mundoeducacao.uol.com.br/geografia/o-que-globalizacao.htm. Acesso em: 19 jul. 2020. 
OLIVEIRA, Fernanda Thaís de; SIMÕES, Wagner Lourenzi. A INDÚSTRIA 4.0 E A PRODUÇÃO NO CONTEXTO DOS ESTUDANTES DA ENGENHARIA. In: SIMPÓSIO DE ENGENHARIA DE PRODUÇÃO - SIENPRO, 2017, Catalão, Goiás, Brasil. A INDÚSTRIA 4.0 E A PRODUÇÃO NO CONTEXTO DOS ESTUDANTES DA ENGENHARIA. Catalão, Goiás, Brasil. 2017. Disponível em: https://files.cercomp.ufg.br/weby/up/1012/o/Fernanda_Tha\%C3\%ADs_de_Oliveira.pdf. Acesso em: 17 jul. 2020.

PSIM. Disponível em: https://powersimtech.com/. Acesso em: 20 jul. 2020.

RASHID, Muhammad H. Eletrônica de Potência: Circuitos, Dispositivos e Aplicações. São Paulo: Makron, 1999.

SANTOS, Amélia Moreira et al. O ENSINO DA ENGENHARIA POR MEIO DE LABORATÓRIOS VIRTUAIS: SOFTWARES DE AUTOMAÇÃO INDUSTRIAL. In: XL CONGRESSO BRASILEIRO DE EDUCAÇÃO EM ENGENHARIA, 2012, Belém-Pa.

Anais. Belém-Pa, 2012. Disponível em: http://www.abenge.org.br/cobenge/arquivos/7/artigos/104258.pdf. Acesso em: 20 jul. 2020

SILVEIRA, Maurício et al. FERRAMENTAS DE SOFTWARE DE ANÁLISE E SÍNTESE DE CIRCUITOS COMO APOIO DIDÁTICO NA MELHORIA DO ENSINO DE ENGENHARIA. In: XXVII CONGRESSO BRASILEIRO DE EDUCAÇÃO EM

ENGENHARIA, 1999, Natal. Anais. Natal, 1999. Disponível em: http://www.abenge.org.br/cobenge/arquivos/20/st/t/t085.pdf._Acesso em: 20 jul. 2020.

\title{
THE USE OF NI MULTISIM LIVE AND PSIM EDUCATIONAL RESOURCES FOR THE INTRODUCTION OF POWER ELECTRONICS SIMULATIONS
}

\begin{abstract}
The growing demand demanded by the labor market in the area of power electronics leads educational institutions to invest in state-of-the-art laboratories, that have very salty prices in the market. To take this discipline through laboratory paths it has been a methodology used for teaching and learning that curricular component, seeking to corroborate the theoretical-practical binomial. Thus, this article deals with the pedagogical strategy of taking the teaching of power electronics to experimental levels, based on simulation software, such as PSIM and NI Multisim, which simulate a laboratory environment,providing its users to simulate possible situations. To this end, the literature review processes followed by simulations and ending with the practices underscore the methodology adopted. Thus, the results indicated a gradual rise in the learning curve of the students who were inserted in this method.
\end{abstract}

Keywords: Power Electronics. Theoretical-practical. Software. Simulation. 